\title{
A new anticoagulation strategy using recombinant human thrombomodulin in patients on veno-venous extracorporeal membrane oxygenation: a retrospective study
}

\author{
Norihisa Yasuda ${ }^{1}$, Koji Goto ${ }^{1}$, Takayuki Mizoguchi ${ }^{2}$, Yoshifumi Ohchi ${ }^{1}$, Shin-Ichi Nureki ${ }^{3}$, \\ Takaaki Kitano ${ }^{1}$
}

${ }^{1}$ Department of Anesthesiology and Intensive Care, Faculty of Medicine, Oita University, Oita, Japan; ${ }^{2}$ Department of Clinical Engineering, Oita University Hospital, Oita, Japan; ${ }^{3}$ Department of Respiratory Medicine and Infectious Diseases, Faculty of Medicine, Oita University, Oita, Japan Contributions: (I) Conception and design: N Yasuda; (II) Administrative support: K Goto; (III) Provision of study materials or patients: Y Ohchi; (IV) Collection and assembly of data: T Mizoguchi; (V) Data analysis and interpretation: SI Nureki, T Kitano; (VI) Manuscript writing: All authors; (VII) Final approval of manuscript: All authors.

Correspondence to: Norihisa Yasuda, MD, PhD. Department of Anesthesiology and Intensive Care, Faculty of Medicine, Oita University, 1-1 Idaigaoka, Hasamamachi, Yufu City, Oita 879-5593, Japan. Email: yasu@oita-u.ac.jp.

Background: During veno-venous extracorporeal membrane oxygenation (VV-ECMO), systemic anticoagulation is required to prevent thrombotic complications within the circuit and oxygenator. The unfractionated heparin (UFH) is commonly administered as a standard anticoagulant, but in our institute recombinant human thrombomodulin (rhTM), instead of UFH, is used as an anticoagulant for VV-ECMO. In the present study, we reviewed whether rhTM could be applied effectively and safely as an anticoagulant agent during VV-ECMO.

Methods: All 15 patients with severe respiratory failure on VV-ECMO were analyzed retrospectively. The following data were collected: age, gender, underlying disease, APACHE-II score, SOFA score, Japanese association for acute medicine (JAAM) DIC score, the usage of anticoagulants, time course of coagulationrelated parameters during ECMO, hemorrhagic and thrombotic complications.

Results: The median age of the patients was 73 years. The median JAAM DIC score at day 0 was 5 points, indicating that 13 patients were diagnosed with DIC at the initiation of VV-ECMO. The total number of days of VV-ECMO runs combined was 193 days, with a median duration of VV-ECMO of 9 days. Among the $15 \mathrm{VV}$-ECMO runs, rhTM was used as monotherapy in 5 runs, and a combination of rhTM and (antithrombin) AT was used in 8 runs. UFH was used in combination with rhTM in only 2 runs. Median ACT and aPTT remained a little longer than normal range over the course of the 14 days of a VV-ECMO run. Bleeding events were observed in 6 cases (40\%) and no major thromboses were observed in all patients.

Conclusions: In this retrospective study, we analyzed 15 patients with severe respiratory failure who were administered rhTM as an anticoagulant during VV-ECMO and found that anticoagulation therapy with rhTM is maybe a feasible option which allows for effective and safe VV-ECMO.

Keywords: Veno-venous extracorporeal membrane oxygenation (VV-ECMO); anticoagulation therapy; recombinant human thrombomodulin (rhTM)

Submitted Jul 27, 2020. Accepted for publication Oct 26, 2020.

doi: 10.21037/apm-20-1487

View this article at: http://dx.doi.org/10.21037/apm-20-1487 


\section{Introduction}

Veno-venous extracorporeal membrane oxygenation (VVECMO) is a rescue therapy for severe respiratory failure which is widely used in many institutes around the world. VV-ECMO has been shown to improve survival in patients with severe acute respiratory distress syndrome (ARDS) in a randomized clinical trial (RCT) (1). It was also used to treat patients with severe respiratory failure during the influenza pandemic of 2009 with a high rescue rate (2). A recent RCT reported that VV-ECMO did not achieve a significant reduction in mortality between the control group and the ECMO group. However, 28\% of the patients in the control group in the RCT crossed over to ECMO for ethical reasons, and post-hoc sensitivity analysis considering the patients crossed over to ECMO in the control group showed a benefit in favor of ECMO group (3).

Technological advances and the accumulation of clinical experience have led to a broader application of VVECMO for patients with severe respiratory failure (4). During VV-ECMO, contact of blood with a nonbiologic surface activates thrombotic and inflammatory responses. Thus, systemic anticoagulation therapy is considered in order to prevent circuit malfunction and thrombosis. The Extracorporeal Life Support Organization (ELSO) guidelines recommend the use of unfractionated heparin (UFH), targeting an activated partial thromboplastin time (aPTT) of 1.5 times the baseline (roughly 50 70 seconds) or an activated clotting time (ACT) of 1.5 times the baseline (180-220 seconds) (5). However, many studies have reported that coagulation tests used to guide anticoagulation therapy are imprecise, and that the targeted ranges of ACT and aPTT during the use of UFH vary markedly among institutes and attending physicians (6-9).

Thrombomodulin (TM) is a physiologic anticoagulant produced in endothelial cells. In Japan, recombinant human soluble TM (rhTM) has been developed and widely used to treat disseminated intravascular coagulation (DIC). rhTM binds directly to thrombin and inactivates it, and a complex of thrombin and rhTM strongly promotes the anticoagulative activity of protein $\mathrm{C}$ (10). Antithrombin (AT) is also frequently used for treating DIC, although supplementing AT during ECMO is not recommend unless the AT-III activity falls below the normal range (5). Critical patients who need VV-ECMO often develop complications including multiple organ failure and coagulopathy such as
DIC. In our institute, when a patient is diagnosed with DIC at the initiation of VV-ECMO, the administration of rhTM is considered as an anticoagulation therapy for both $\mathrm{VV}$ ECMO and DIC. In the present study, we retrospectively assessed the acceptability of rhTM as an anticoagulant for use during VV-ECMO.

We present the following article in accordance with the STROBE reporting checklist (available at http://dx.doi. org/10.21037/apm-20-1487) (11).

\section{Methods}

The present study was approved by the institutional review board of the Medical Faculty of Oita University (No. 1461) and the need for informed consent was waived. This study was conducted in accordance with the Declaration of Helsinki (as revised in 2013). We received no specific grant from any funding agency. Patients with severe respiratory failure who received VV-ECMO were selected from a database of patients admitted to the intensive care unit of Oita University Hospital between January 2012 and December 2017. Exclusion criteria were age under 18 years, no administration of rhTM during VVECMO, and use of other mechanical circulatory support devices [e.g., left ventricular assist device (LVAD)]. In all patients, VV-ECMO was initiated as a rescue therapy to treat hypoxia under conventional mechanical ventilation according to ELSO guidelines (5). Patients who received renal replacement therapy (RRT) were also included in the present study.

rhTM was administered during $\mathrm{VV}$-ECMO in patients diagnosed with DIC [i.e., Japanese Association for Acute Medicine (JAAM) DIC score $\geq 4$ points] (Table 1) (12). Antithrombin (AT) was administered when AT-III activity fell below $70 \%$. Dosages of rhTM and AT were determined according to the manufacturers' recommendations.

VV-ECMO was initiated by passing two NSH heparincoated cannulas (PCKC-V and PCKC-A; Senko Medical Instrument Manufacturing Co. Ltd., Tokyo, Japan) through the femoral vein (22-24 Fr) and internal jugular vein (16$18 \mathrm{Fr}$ ). The blood flow of $\mathrm{VV}$-ECMO was maintained at $\geq 2.5 \mathrm{~L} / \mathrm{min}$. Data were obtained at days $0,3,7,10$, and 14 from the database and included platelet counts, prothrombin time-international normalized ratio (PT-INR), aPTT, ATIII activity, fibrin/fibrinogen degradation products (FDP), plasmin- $\alpha 2$-plasmin inhibitor complex (PIC), thrombinantithrombin complex (TAT), and ACT. We also collected 
Table 1 JAAM DIC scoring system

\begin{tabular}{lc}
\hline Variables & Score \\
\hline Systemic inflammatory response syndrome criteria & \\
$\geq 3$ & 1 \\
$0-2$ & 0 \\
Platelet counts $\left(\times 10^{4} / \mu \mathrm{L}\right)$ & 3 \\
$<8.0$, or a more than $50 \%$ decrease within 24 hours & 1 \\
$\geq 8.0,<12.0$ or a more than $30 \%$ decrease within & \\
24 hours & 0 \\
$\geq 12.0$ & \\
PT-INR & 1 \\
$\geq 1.2$ & 0 \\
$<1.2$ & \\
FDP $(\mu \mathrm{g} / \mathrm{mL})$ & 3 \\
$\geq 25$ & 1 \\
$10-<25$ & 0 \\
$<10$ & \\
\hline
\end{tabular}

4 points or more, diagnosed as DIC. JAAM, Japanese Association for Acute Medicine; DIC, disseminated intravascular coagulation; PT-INR, prothrombin time-international normalized ratio; FDP, fibrin/fibrinogen degradation products.

data regarding dosages and administration durations of rhTM, AT, and UFH during VV-ECMO; calculated Acute Physiology and Chronic Health Evaluation-II (APACHE-II) scores, Sequential Organ Failure Assessment (SOFA) scores, Murray scores, and JAAM DIC scores at day 0. Missing values were scored as 0 . We also recorded and analyzed hemorrhagic and thrombotic complications during $\mathrm{VV}$ ECMO.

A high hemoglobin target of $12-14 \mathrm{~g} / \mathrm{dL}$ and platelet count target of more than $50,000 / \mathrm{mm}^{3}$ were used to optimize oxygen delivery and prevent hemorrhagic events. Transfusion of packed red blood cell and platelet concentrates was performed at the discretion of attending physicians.

\section{Statistical analysis}

Data are presented as median and interquartile ratio (IQR) or numbers, as appropriate. Differences in each coagulation-related parameter between days $0,3,7,10$ and 14 were evaluated using the Friedman test. $\mathrm{P}<0.05$ was considered statistically significant. Statistical analysis was carried out using Statflex Statistical Software version 7.0 (Artech Co, Ltd., Osaka, Japan).

\section{Results}

A total of 18 serial VV-ECMO runs in 18 patients were identified between January 2013 and December 2017. Of these, 15 runs in 15 patients were analyzed after excluding 2 patients with LVAD use and 1 patient who was not administered rhTM during VV-ECMO.

Baseline characteristics are summarized in Table 2. Median age of patients was 73 years. All patients suffered from severe respiratory failure with high Murray scores (median: 3 points). Median APACHE-II and SOFA scores at day 0 were 28 and 11 points, respectively, suggesting that multiple organs were impaired in all the patients at the time of VV-ECMO initiation. The median JAAM DIC score at day 0 was 5 points, indicating that 13 patients were diagnosed with DIC at the time of VV-ECMO initiation. The remaining 2 patients also met the DIC criteria after the start of ECMO.

The total number of days of all VV-ECMO runs combined was 193 days, with a median duration of VVECMO of 9 days (IQR: 7-18). The maximum and minimal durations of VV-ECMO were 22 and 6 days, respectively. Continuous renal replacement therapy (CRRT) was performed in all patients at some point during VV-ECMO to treat renal dysfunction. All patients were followed up to the termination of VV-ECMO and the maximum follow-up day was 22 days.

\section{Anticoagulation therapy}

Among the $15 \mathrm{VV}$-ECMO runs, rhTM was used as monotherapy in 5 runs, and a combination of rhTM and AT was used in 8 runs. UFH was administered in combination with rhTM in only 2 runs (Table 3). The median dosage of rhTM was 130 units/kg/day. During the combined total of $193 \mathrm{VV}$-ECMO days, rhTM was used for 139 days but not for the remaining 54 days due to bleeding events or lack of DIC. The dosage of AT was 1,500 units/day, and the total number of days of administration was 45 days. UFH was administered for only 13 days (Table 4). Nafamostat mesilate (NM) was administered at a dosage of $20-30 \mathrm{mg}$ /hour while CRRT was performed as renal support therapy. 
Table 2 Baseline characteristics

\begin{tabular}{|c|c|}
\hline Variables & Values (total $n=15$ ) \\
\hline Age (year) & 73 (69 to 77$)$ \\
\hline Gender (male/female), $\mathrm{n}$ & $10 / 5$ \\
\hline APACHE II score at day 0 & 28 (25 to 31$)$ \\
\hline SOFA score at day 0 & 11 (10 to 13$)$ \\
\hline Murray score at day 0 & 3 (3 to 3.27$)$ \\
\hline JAAM DIC score at day 0 & 5 (4 to 6$)$ \\
\hline \multicolumn{2}{|l|}{ Baseline disease } \\
\hline Interstitial pneumonia, $\mathrm{n}$ & 7 \\
\hline Aspiration pneumonia, $\mathrm{n}$ & 2 \\
\hline Pneumocystis pneumonia, $\mathrm{n}$ & 2 \\
\hline Septic ARDS, $n$ & 2 \\
\hline Drug-induced pneumonia, $\mathrm{n}$ & 1 \\
\hline Fungal pneumonia, n & 1 \\
\hline ECMO days/patient & 9 (7 to 18$)$ \\
\hline Total ECMO days & 193 \\
\hline Days in ICU & 14 (9 to 23$)$ \\
\hline ICU survival, $\mathrm{n}$ & 6 \\
\hline Hospital survival, $n$ & 4 \\
\hline CRRT (yes/no) & $18 / 0$ \\
\hline
\end{tabular}

Values are presented as median (25th/75th percentile). $n$, numbers; APACHE-II, Acute Physiology and Chronic Health Evaluation-II; SOFA, Sequential Organ Failure Assessment; JAAM DIC, Japanese Association for Acute Medicine-DIC score; ARDS, Acute respiratory distress syndrome; ECMO, Extracorporeal membrane oxygenation; CRRT, Continuous renal replacement therapy.

Table 3 Anticoagulation therapy on VV-ECMO

\begin{tabular}{lc}
\hline Anticoagulant & $\mathrm{n}$ \\
\hline rhTM only & 5 \\
rhTM + AT & 7 \\
rhTM + UFH & 1 \\
rhTM + AT + UFH & 2 \\
Total & 15 \\
\hline
\end{tabular}

VV-ECMO, veno-venous extracorporeal membrane oxygenation; rhTM, recombinant human thrombomodulin; AT, antithrombin; UFH, unfractionated heparin.

\section{Coagulation-related parameters}

As mentioned above, JAAM DIC scores were $\geq 4$ points in 13 patients at the time of $\mathrm{VV}$-ECMO initiation, and decreased platelet counts, prolonged PT-INR, and increased FDP were observed at day 0. According to ELSO guidelines, the use of UFH is recommended to maintain $\mathrm{ACT}$ or $\mathrm{aPTT}$ at 1.5 times the normal value. In the present study, median ACT and aPTT remained within the ranges of 138-158 seconds and 41.1-48.2 seconds, respectively, over the course of the 14 days of a VV-ECMO run, a little longer than normal range, respectively. FDP levels did not change significantly over time, suggesting that no major thromboses were formed in the VV-ECMO circuit or in patients. Other parameters also showed no significant changes throughout the course of VV-ECMO (Table 5). We had no missing values of the variables in all patients during VV-ECMO.

\section{Hemorrbagic and thrombotic events}

Bleeding complications were observed in 6 patients (40\%) (Table 6). Severe intracranial hemorrhage occurred in 1 of 2 patients who were administered rhTM and UFH concurrently, leading to discontinuation of VV-ECMO. Gastrointestinal bleeding occurred in 2 patients who were simultaneously administered rhTM and AT, but bleeding was stanched endoscopically in both cases. In cases where bleeding occurred, anticoagulants were discontinued temporarily as long as the ECMO flow was maintained, and restarted after hemostasis was confirmed. The VV-ECMO circuit and oxygenator were visually inspected on a daily basis, but no major thrombosis formation was observed during all $15 \mathrm{VV}$-ECMO runs.

\section{Discussion}

In this retrospective study, we demonstrated that rhTM is an acceptable anticoagulant for patients on VV-ECMO and can be used as an alternative to UFH. To the best of our knowledge, this is the first study to report on the use of rhTM during VV-ECMO. Moreover, the incidence of bleeding complications was not as high as those reported previously, and there were no cases of evident thrombosis. ELSO guidelines recommend that UFH be considered as an anticoagulant during VV-ECMO (5). UFH, the most 
Table 4 Dosages and days of anticoagulant administration during VV-ECMO

\begin{tabular}{lcc}
\hline Anticoagulant (total: 193 days) & Dosages/day & Days of administration \\
\hline rhTM & $130(130-380)$ units/kg & 139 \\
AT & 1,500 units & 45 \\
UFH & $10,000(5,000-12,500)$ & 13 \\
\hline
\end{tabular}

VV-ECMO, veno-venous extracorporeal membrane oxygenation; rhTM, recombinant human thrombomodulin; AT, antithrombin; UFH, unfractionated heparin.

Table 5 Changes over time of coagulation-related parameters for VV-ECMO

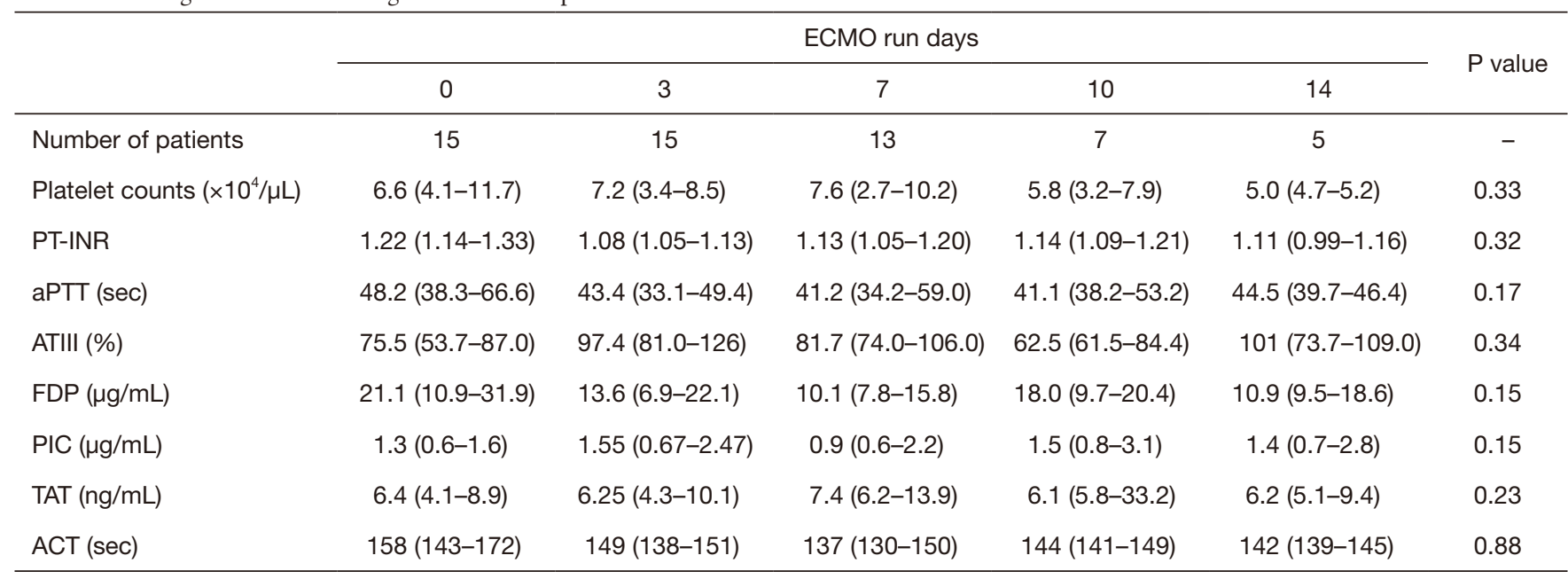

VV-ECMO, veno-venous extracorporeal membrane oxygenation; PT-INR, prothrombin time-international normalized ratio; aPTT, activated partial thromboplastin time; AT-III, antithrombin III; FDP, fibrinogen/fibrin degradation products; PIC, plasmin- $\alpha 2-$ plasmin inhibitor complex; TAT, thrombin-antithrombin complex; ACT, activated clotting time.

Table 6 Bleeding complications during VV-ECMO

\begin{tabular}{lccc}
\hline \multirow{2}{*}{ Site of bleeding } & \multicolumn{3}{c}{ Anticoagulation therapy } \\
\cline { 2 - 4 } & rhTM only & rhTM + AT & rhTM + UFH \\
\hline Intracranial, $\mathrm{n}$ & - & - & 1 \\
Gastrointestinal, $\mathrm{n}$ & - & 2 & 1 \\
Surgical site, $\mathrm{n}$ & 1 & - & 1 \\
\hline
\end{tabular}

VV-ECMO, veno-venous extracorporeal membrane oxygenation; rhTM, recombinant human thrombomodulin; AT, antithrombin; $\mathrm{UFH}$, unfractionated heparin.

common anticoagulant used in patients on VV-ECMO worldwide, can be titrated easily and reversed when necessary. According to ELSO guidelines, UFH therapy should target an ACT of 1.5 times the normal value (roughly 180-220 seconds) (5). However, ACT was reported to be influenced by hemodilution and thrombocytopenia, and low dosages of UFH did not correlate with ACT and
aPTT (6). Moreover, target values of coagulation variables, as well as their dosages in anticoagulation therapy, vary among institutes (7-9).

The results of the present study demonstrate that anticoagulation therapy using rhTM on VV-ECMO is acceptable in clinical settings. Bleeding complications were comparable with those observed in previous studies, and increases in FDP were small throughout the course of VVECMO, suggesting that no major thromboses formed during anticoagulation therapy.

rhTM was developed in Japan and has been widely used as an anticoagulant to manage DIC since 2008. It binds directly to and inactivates thrombin. Moreover, a complex of thrombin and rhTM strongly promotes the anticoagulative activity of protein C. A phase III RCT of rhTM demonstrated that rhTM can significantly improve DIC and alleviate bleeding symptoms in DIC patients, compared with UFH (10). Hayakawa et al. reported that the 
administration of rhTM significantly reduced mortality in patients with septic DIC (13). Currently, a phase III RCT is being conducted worldwide to evaluate the efficacy of rhTM in patients with sepsis and DIC, although a recent study found no effect of rhTM on mortality (14).

In the present study, AT was administered in combination with rhTM in 9 patients $(60 \%)$. ELSO guidelines suggest that AT-III activity be maintained within the normal range when UFH is administered, as the effect of UFH may otherwise be reduced (5). AT is also frequently used for treating DIC. In an RCT of AT in patients with severe sepsis, although the overall analysis failed to detect a beneficial effect of AT therapy, a subgroup analysis of patients treated without concomitant UFH showed a significant reduction in mortality (15). Gando et al. reported that, relative to the control group, AT therapy improved recovery rates from DIC (16). In Japan, the use of AT is considered when AT-III activity falls below $70 \%$; 9 patients in the present study met this criterion and were administered AT for 5 days in accordance with the manufacturer's instructions.

In the present study, UFH was used only in 3 of 15 patients (20\%) during VV-ECMO. In our institute, VV$\mathrm{ECMO}$ has been performed for severe respiratory failure since 2013. Initially, UHF was used as the standard anticoagulant in accordance with ELSO guidelines, with additional anticoagulants (e.g., rhTM and AT) administered when patients were diagnosed with DIC. However, 1 patient developed severe brain hemorrhage as a complication of VV-ECMO with UFH and rhTM, requiring termination of VV-ECMO. Since that incident, our institute has stopped using UFH as an initial anticoagulant for VV-ECMO when patients meet the JAAM DIC criteria; these patients are instead administered rhTM for DIC throughout the course of VV-ECMO.

The lack of laboratory targets to evaluate the adequacy of anticoagulation, such as ACT and aPTT in the case of $\mathrm{UFH}$, is a concern when using rhTM with VV-ECMO. rhTM does not directly inhibit coagulation factors and has little influence on ACT and aPTT. Thus, these laboratory variables are not useful for confirming the adequacy of anticoagulation. We carefully monitor thrombus formation in the VV-ECMO circuit and signs of thrombus formation in patients, such as elevated FDP. In the present study, however, none of the patients showed a marked increase in FDP levels during the course of VV-ECMO, and there was no evidence of major thrombus formation in the VVECMO circuit.
Another concern is that the half-life of rhTM is 20 hours, and no reversal drugs, such as protamine for UFH, have been developed (17). Therefore, we anticipated a difficulty in controlling bleeding complications. Bleeding complications were noted in 6 of the $15 \mathrm{VV}$-ECMO runs $(40.0 \%)$. Among the complications was severe intracranial hemorrhage in a patient who was administered UFH in combination with rhTM. The combination of rhTM and UFH may increase bleeding risk, but the risk of these anticoagulants administered together was not assessed in the present study due to the limited sample size. Other cases of bleeding were managed by stanching and discontinuation of anticoagulants, allowing for continuation of VV-ECMO. Bleeding and thrombotic complications on ECMO are common and have a significant impact on patient outcomes (18). Combes et al. reported that bleeding events occurred in $46 \%$ of the patients with VV-ECMO (3). One meta-analysis that reviewed 12 studies with 1,763 adult patients on ECMO found a bleeding complication rate of $33 \%$ (19). In a large retrospective review of 21,845 pediatric cardiac patients requiring ECMO, hemorrhagic complications occurred in 8,480 patients (39\%) (20). Panigada et al. reported that 3 patients (25\%) complicated major bleeding, which was lower than $40 \%$ in our results. However, the mean platelet counts in their study were relatively high during $\mathrm{VV}$ ECMO, compared with our results (21). Similar to their results, there were no significant differences on clinical data between bleeding group and no-bleeding group in the present study. In the database of ELSO, incidence rates of cannulation-site, surgical-site, gastrointestinal, and cerebral hemorrhagic events in adults with respiratory failure were $14 \%, 11 \%, 6 \%$, and $4 \%$, respectively (22). In our patients, while no evident bleeding events were observed at the cannulation site, and bleeding at surgical sites was noted in only 1 patient, the rate of gastrointestinal bleeding events was relatively high. The bleeding complication rate $(40 \%)$ in the present study seems to be similar to the previous studies reported, however our sample size is so limited that the bleeding risk of rhTM during VV-ECMO must be further evaluated by accumulating more data. In our patients, no evident thrombotic events were found, but Rastan et al. showed there is a high rate of clinically unrecognized venous thromboembolism and systemic thromboembolic events, suggesting that the incidence of thrombotic events in ECMO may be underreported when relying on clinical evaluation alone (23). CRRT was performed at some point during all VV-ECMO runs. 
During CRRT, NM was administered in the CRRT circuit as an anticoagulant. Given that the half-life of NM is reported to be roughly 5-8 minutes (24), the possibility of NM influencing the systemic anticoagulative effect cannot be completely excluded.

Our results suggest that rhTM may also provide an option for anticoagulation management in patients with heparin-induced thrombocytopenia (HIT) or heparin resistance. In patients with HIT, anticoagulation therapy is usually performed using argatroban or bivalirudin (8). rhTM can be considered a potential alternative in anticoagulation therapy during ECMO in this patient population.

The present study has some limitations worth noting. First, this study used a single-center retrospective design, and unknown confounders may have affected our results. Second, large-scale, prospective RCTs will be needed to confirm that rhTM is an acceptable anticoagulant for VVECMO. Third, since we did not examine the volume of transfusion during VV-ECMO, the impact of transfusion rates on bleeding events is unclear. Finally, in recent years, the complication of venous thrombosis after VV-ECMO has been reported (25). Although none of our patients showed markedly elevated FDP levels, we did not screen for thrombus formation after VV-ECMO by contrastenhanced computed tomography (CT). It is not clear that our results can be applied to other institutes or patients with other underlying diseases. The present study should be considered as a preliminary report, thus further randomized controlled studies are needed to confirm the acceptability and safety of rhTM on VV-ECMO in place of UFH.

\section{Conclusions}

The present study analyzed 15 patients with severe respiratory failure who were administered rhTM as an anticoagulant during VV-ECMO and found that anticoagulation therapy with rhTM is an acceptable option which allows for effective and safe VV-ECMO.

\section{Acknowledgments}

Funding: None.

\section{Footnote}

Reporting Checklist: The authors have completed the STROBE reporting checklist. Available at http://dx.doi. org/10.21037/apm-20-1487

Data Sharing Statement: Available at http://dx.doi. org/10.21037/apm-20-1487

Conflicts of Interest: All authors have completed the ICMJE uniform disclosure form (available at http://dx.doi. org/10.21037/apm-20-1487). The authors have no conflicts of interest to declare.

Ethical Statement: The authors are accountable for all respects of the work in ensuring that questions related to the accuracy or integrity of any part of the work are appropriately investigated and resolved. This retrospective study was approved by the institutional ethics board of the Medical Faculty of Oita University (No. 1461) and the need for informed consent was waived. This study was conducted in accordance with the Declaration of Helsinki (as revised in 2013).

Open Access Statement: This is an Open Access article distributed in accordance with the Creative Commons Attribution-NonCommercial-NoDerivs 4.0 International License (CC BY-NC-ND 4.0), which permits the noncommercial replication and distribution of the article with the strict proviso that no changes or edits are made and the original work is properly cited (including links to both the formal publication through the relevant DOI and the license). See: https://creativecommons.org/licenses/by-nc-nd/4.0/.

\section{References}

1. Peek GJ, Mugford M, Tiruvoipati R, et al. Efficacy and economic assessment of conventional ventilatory support versus extracorporeal membrane oxygenation for severe adult respiratory failure (CESAR): a multicenter randomized controlled trial. Lancet 2009;374:1351-63.

2. Noah MA, Peek GJ, Finney SJ, et al. Referral to an extracorporeal membrane oxygenation center and mortality among patients with severe 2009 influenza A (H1N1). JAMA 2011;306:1659-68.

3. Combes A, Hajage D, Capellier G, et al. Extracorporeal Membrane Oxygenation for Severe Acute Respiratory Distress Syndrome. N Engl J Med 2018;378:1965-75.

4. Sen A, Callisen HE, Alwardt CM, et al. Adult venovenous extracorporeal membrane oxygenation for severe respiratory failure: Current status and future per spectives. Ann Card Anaesth 2016;19:97-111.

5. Extracorporeal Life Support Organization: ELSO 
guidelines for adult respiratory failure. Version 1.4. August 2017. Available online: www.elso.org/Portals/0/ELSO Guidelines General All ECLS Version 1_4.pdf

6. Esper SA, Levy JH, Waters JH, et al. Extracorporeal membrane oxygenation in the adult: a review of anticoagulation monitoring and transfusion. Anesth Analg 2014;118:731-43.

7. Bembea MM, Annich G, Rycus P, et al. Variability in anticoagulation management of patients on extracorporeal membrane oxygenation: an international survey. Pediatr Crit Care Med 2013;14:e77-84.

8. Sklar MC, Sy E, Lequier L, et al. Anticoagulation practice during venovenous extracorporeal membrane oxygenation for respiratory failure, A sistematic review. Ann Am Thorac Soc 2016;13:2242-50.

9. Raiten JM, Wong ZZ, Spelde A, et al. Anticoagulation and transfusion therapy in patients requiring extracorporeal membrane oxygenation. J Cardiothorac Vasc Anesth 2017;31:1051-9.

10. Saito H, Maruyama I, Shimazaki S, et al. Efficacy and safety of recombinant human soluble thrombomodulin (ART-123) in disseminated intravascular coagulation: results of a phase III, randomized, double-blind clinical trial. J Thromb Haemost 2007;5:31-41.

11. von Elm E, Altman DG, Egger M, et al. The strengthening the reporting of observational studies in epidemiology (STROBE) statement: Guidelines for reporting observational studies. PLoS Med 2007;4:e296.

12. Gando S, Iba T, Eguchi Y, et al. A multicenter, prospective validation of disseminated intravascular coagulation diagnostic criteria for critically ill patients: results of a multicenter, prospective survey. Crit Care Med 2006;34:625-31.

13. Hayakawa M, Yamakawa K, Saito S, et al. Recombinant human soluble thrombomodulin and mortality in sepsis-induced disseminated intravascular coagulation. A multicentre retrospective study. Thromb Haemost 2016;115:1157-66.

14. Vincent JL, Francois B, Zabolotskikh I, et al. Effect of a Recombinant Human Soluble Thrombomodulin on Mortality in Patients With Sepsis-Associated Coagulopathy: The SCARLET Randomized Clinical Trial. JAMA 2019;321:1993-2002.

15. Kienast J, Juers M, Wiedermann CJ, et al. Treatment effects of high-dose antithrombin without concomitant heparin in patients with severe sepsis with or without disseminated intravascular coagulation. J Thromb Haemost 2006;4:90-7.
16. Gando S, Saitoh D, Ishikura H, et al. A randomized, controlled, multicenter trial of the effects of antithrombin on disseminated intravascular coagulation in patients with sepsis. Crit Care 2013;17:R297.

17. Nakashima M, Uematsu T, Umemura K, et al. A novel recombinant soluble human thrombomodulin, ART-123, activates the protein $\mathrm{C}$ pathway in healthy male volunteers. J Clin Pharmacol 1998;38:540-4.

18. Thiagarajan RR, Barbaro RP, Rycus PT, et al. Extracorporeal life support organization registry international report 2016. ASAIO J 2017;63:60-7.

19. Zangrillo A, Landoni G, Biondi-Zoccai G, et al. A metaanalysis of complications and mortality of extracorporeal membrane oxygenation. Crit Care Resusc 2013;15:172-8.

20. Werho DK, Pasquali SK, Yu S, et al. Hemorrhagic complications in padiatric cardiac patients on extracorporeal membrane oxygenation: an analysis of the Extracorporeal Life Support Organization Registry. Pediatr Crit Care Med 2015;16:276-88.

21. Panigada M, Artoni A, Passamonti SM, et al. Hemostasis changes during veno-venous extracorporeal membrane oxygenation for respiratory support in adults. Minerva Anestesiol 2016;82:170-9.

22. Conrad SA, Rycus PT, Dalton H. Extracorporeal life support Registry Report 2004. ASAIO J 2005;51:4-10.

23. Rastan AJ, Lachmann N, Walther T, et al. Autopsy findings in patients on postcardiotmy extracorporeal membrane oxygenation. Int J Artif organs 2006;29:1121-31.

24. Nakae H, Tajimi K. Pharmacokinetics of Nafamostat Mesilate During Continuous Hemodiafiltration with a Polyacrylonitrile Membrane. Ther Apher Dial 2003;7:483-5.

25. Fisser C, Reichenbächer C, Müller T, et al. Incidence and risk factors for cannula-related venous thrombosis after venovenous extracorporeal membrane oxygenation in adult patients with acute respiratory failure. Crit Care Med 2019;47:e332-9.

Cite this article as: Yasuda N, Goto K, Mizoguchi T, Ohchi Y, Nureki SI, Kitano T. A new anticoagulation strategy using recombinant human thrombomodulin in patients on venovenous extracorporeal membrane oxygenation: a retrospective study. Ann Palliat Med 2021;10(2):1834-1841. doi: 10.21037/apm20-1487 